Original Research ARTicle

\title{
Flame Retardant Effect of Isocyanate Trimer on Polyisocyanurate Foam
}

\author{
Mingyang Du,Chunze He,Canhui Zhou \\ School of Materials Science and Engineering, Chengdu Jiaotong University, Sichuan, China
}

\begin{abstract}
The effect of Isocyanate trimer on the flame retardancy mechanism of polyisocyanurate (PIR) foam was studied through 3 aspects including the mechanism of thermal decomposition stage in condensed phase, the barrier mechanism of carbon layer formation on the surface in condensed phase and the mechanism in gaseous phase by using infrared spectroscopy, scanning electron microscopy, thermal analysis, cone calorimeter, high temperature pyrolysis gas chromatography mass spectrometry. The results show that the trimer can improve the thermal stability of the PIR foam and is not easily decomposed in the combustion. The trimer can increase the carbon content of the PIR foam to $29.9 \%$ than that of the polyurethane (PU) foam. The carbon layer formed is denser, and it can retards heat and oxygen and thus improve the flame retardant properties; trimer can reduce the release of flammable polyol gas, decompose into more carbon dioxide which is inert gas and it has certain flame retardant effect in the gaseous phase.
\end{abstract}

KEYWORDS: Polyisocyanurate foam; Isocyanate trimer; Flame retardant mechanism

Polyisocyanurate Foam (PIR) is a new type of insulation material used in building construction. Due to the introduction of the isocyanate trimer structure in the foam molecular structure, the temperature and flame resistance of the foam can be improved, in which its thermal stability and flame retardancy are higher than that of the PU foam [1,2].

On the flame retardant mechanism of polymers, the classical flame retardant theory is based on the combustion process designed which including the following aspects: the mechanism of thermal decomposition stage in condensed phase, the barrier mechanism of carbon layer formation on the surface in condensed phase and the mechanism in gaseous phase [3]. Zhan et al. [4,5], Kulesza et al. [6] and Kunze et al. [7] determined the thermal decomposition characteristic of polyurethane (PU) foam at different temperatures and qualitatively analyzed the entire thermal decomposition process of PU by using Thermogravimetric Analysis and Fourier Transform Infrared Spectroscopy (TG-FTIR), thermogravimetric analysis and mass spectrometry (TG-MS) and Pyrolysis gas chromatography mass spectrometry (Py/GC-MS). By using TG and SEM characterization, Hu et al. [8] found that the addition of expandable graphite and dimethyl phosphonate in PIR-PU foam caused its surface carbon layer structure became denser and the thermal stability of the material was increased after combustion. Gao et al. [9] studied the synergistic effect of expandable graphite, ethyl diethyl phosphonate and organic modified montmorillonite by using a cone-shaped calorimeter (CONE) and it shows that the flame retardant property of PIR foam increased, this is due to the formula can promote the formation of carbon layer in which can isolate oxygen and heat effectively.

As a reactive flame retardant structure, the trimer structure has a great effect on the flame retardancy of PIR foam, but the study on the mechanism of its action is still not completely. In this paper, the effect of Isocyanate trimer on the flame retardancy mechanism of polyisocyanurate (PIR) foam was studied through 3 aspects including the mechanism of thermal decomposition stage in condensed phase, the barrier mechanism of carbon layer formation on the surface in condensed phase and the mechanism in gaseous phase by using infrared spectroscopy, scanning electron microscopy, thermal analysis, cone calorimeter, high temperature pyrolysis gas chromatography mass spectrometry.

Copyright (C) 2017 -. This is an Open Access article distributed under the terms of the Creative Commons Attribution-NonCommercial 4.0 International License (http://creativecommons.org/licenses/by-nc/4.0/), permitting all non-commercial use, distribution, and reproduction in any medium, provided the original work is properly cited. 


\section{Experimental part}

\subsection{Experimental materials}

Polyester Polyol (PS-3152): Industrial grade, Nanjing Jinling Steinpan Chemical Co., Ltd.; Diphenylmethane diisocyanate (Desmodur44V20L): Industrial grade, Kesiqi Polymer (China) Co., Ltd. Silicone stabilizer ( L-6920): Industrial grade, supplied by Kesi Polymer (China) Co., Ltd.; N, N-Dimethylcyclohexylamine (POLYCAT-8): Industrial Grade, Jiangdu Dajiang Chemical Plant; (PU-1792): industrial grade, Jiangdu Dajiang Chemical Plant; HCFC-141b (hydrochlorofluorocarbon-141b): industrial grade, industrial grade, Changshu three love rich fluoride Chemical Co., Ltd..

\subsection{Foam test recipe and sample preparation}

Due to many elements, the approved design was three months later than planned. Half time has been used for design and construction preparation so the time pressure is very big for the project, how EPC contractor can completes the project is one of the key tasks in run over the project.

Experimental used Foam systems and Foam formulations as shown in Tab. 1

\begin{tabular}{|l|l|l|}
\hline Component & PU & PIR \\
\hline 44V20L (index) & 110 & 350 \\
\hline PS-3152 & 100 & 100 \\
\hline PC-5 & 0.3 & 0.3 \\
\hline Water & 0.5 & 0.5 \\
\hline HFC-141B & 15 & 20 \\
\hline L6920 & 4 & 4 \\
\hline PC-8 & 1.5 & 0 \\
\hline PU-1792 & 0 & 5 \\
\hline
\end{tabular}

Tab. 1 Foaming formulation of PIR and PU foam

Polyurethane foam preparation: refer to Tab. 1 This study used two foam systems, one group as a reference sample PU foam, the other group is PIR foam, two kinds of foam are not added flame retardant. The preparation of the foam has two main processes: Firstly, polyol, catalyst, blowing agent and the surfactant are mixed and stirred at room temperature to obtain a mixture called white matter (if in the summer, it should be stored into the refrigerator, the control temperature is $20^{\circ} \mathrm{C}$ ); secondly, isocyanate is quickly added to the white material, stir in high-speed for 6s and then quickly put into the mold $(150 \mathrm{~mm} \times 150 \mathrm{~mm} \times 100 \mathrm{~mm})$, observe the characteristics of foam reaction, record the duration of white matter formation, gel formation and foam formation by using stopwatch. At the end, the foam become hardened and recovered to room temperature, then the foam is placed into the oven at $80{ }^{\circ} \mathrm{C}$ for 24 hours.

\subsection{Testing and characterization}

\subsubsection{Thermogravimetric analysis (TGA):}

NETZSCH-STA449C thermogravimetric analyzer (German resistant company), the heating rate of $20{ }^{\circ} \mathrm{C} / \mathrm{min}$, the temperature range of $20 \sim 800{ }^{\circ} \mathrm{C}$, air atmosphere.

\subsubsection{Infrared Spectroscopy (FT-IR):}

EQUINOXSS / HYPERION 2000 Infrared Spectrometer (Germany BRUKER Spectrometer), spectral range 7500 $370 \mathrm{~cm}-1$, resolution $<0.5 \mathrm{~cm}-1$. High temperature infrared preparation: the foam sample placed in the muffle furnace for 5 mins at the set temperature, and then the sample is undergo infrared testing.

\subsubsection{Pyrolysis gas chromatography mass spectrometry (Py / GC-MS) analysis:}

QP-2010 Ultra type pyrolysis gas chromatography mass spectrometry (Shimadzu Corporation), the mass range (m/ z) of $1.5 \sim 1090$, The pyrolysis temperature ranges from 350 to $750^{\circ} \mathrm{C}$. 


\subsubsection{Scanning Electron Microscope:}

S-2360N Scanning Electron Microscope (Hitachi, Japan) The desired sample is the residual carbon residue after the foam is measured by the cone calorimeter. Before the observation, it is necessary to cut the section and then spray the gold.

\subsubsection{Limit oxygen index determination:}

HC-2A limit oxygen index instrument (Nanjing Jiangning Analytical Instrument Co, Ltd.) in accordance with GB / T 2406 test, the sample size is $120 \mathrm{~mm}$ long, $10 \mathrm{~mm}$ wide, $4 \mathrm{~mm}$ thick, working pressure of $0.1 \mathrm{MPa}$, The timer accuracy is not less than $0.1 \mathrm{~s}$, igniter flame length $(5 \sim 50) \mathrm{mm}$ can be adjusted, the purity of gas source is not less than $98 \%$ of the oxygen and nitrogen.

\subsubsection{Cone calorimeter test:}

The use of the United Kingdom FTT's FTT0007 cone calorimeter is to conduct a real combustion behavior assessment on foam, that is, to determine the amount of smoke, mass loss rate, oxygen consumption analysis, $\mathrm{CO} 2$ and heat release rate. Thermal radiation power of $50 \mathrm{~kW} / \mathrm{m} 2$; sample size of $100 \mathrm{~mm} \times 100 \mathrm{~mm} \times 25 \mathrm{~mm}$; implementation of the standard GB / T 16172 or ISO 5660-1. RSR is the smoke release rate, $\mathrm{m} 2$ / (s $\cdot \mathrm{m}-2)$; TSR is the total amount of smoke released, $\mathrm{m} 2 / \mathrm{m} 2 ; \mathrm{HRR}$ is the heat release rate, $\mathrm{kW} / \mathrm{m} 2$; THR is the total heat release, $\mathrm{kJ} / \mathrm{m} 2$.

\section{Results and discussion}

\subsection{Combustion model}

Through the comprehensive analysis of the literature, the polyurethane combustion flow chart is shown in Fig. 1 [3]. It is aimed at judging the mechanism of flame retardant by its combustion process. In Part 1, the thermal decomposition process in condensed phase of polyurethane foam is mainly the process where the thermal decomposition products to break through the carbon layer into the gaseous phase. In Part 2, the combustible gas in the gas phase undergo combustion and the inert gas release; In Part 3, the surface of the carbon layer, some thermosetting plastics in the combustion will produce surface carbon layer, and the surface carbon layer has flame retardant effect.

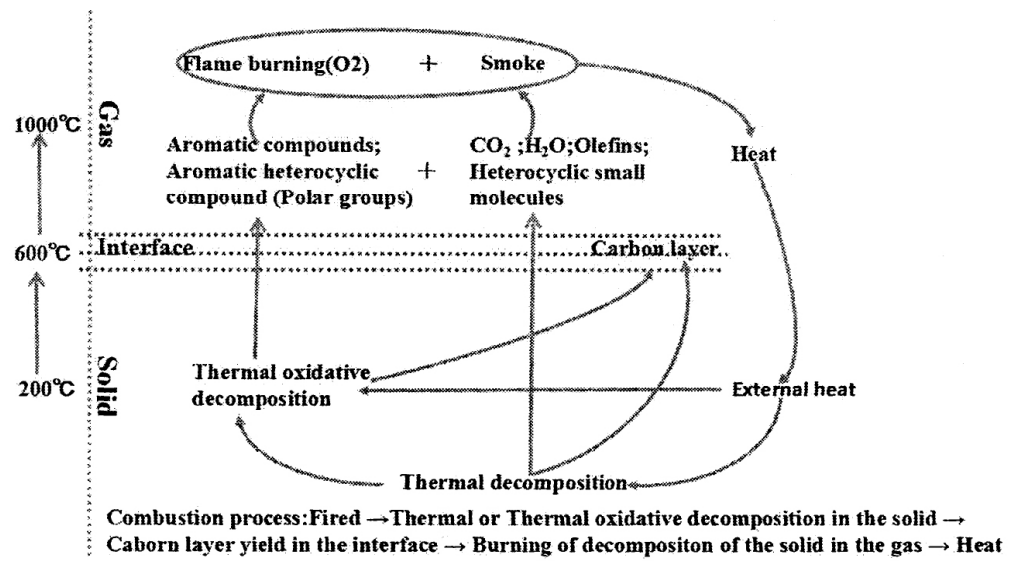

Fig. 1

Therefore, the flame retardant mechanism of the trimer structure on the PIR foam is studied from the mechanism of thermal decomposition stage in condensed phase, the barrier mechanism of carbon layer formation on the surface in condensed phase and the mechanism in gaseous phase.

\subsection{PU and PIR foam combustion performance comparison}

The PU used as the blank control group in order to study the effect of trimer structure on the flame retardancy of PIR foam, the changes of the flame retardant in foam was tested in the present of trimer. The infrared characteristic peak of the trimeric structure is usually located at about $1410 \mathrm{~cm}-1$ [10] Fig. 2 shows the infrared spectrum of PIR foam and PU foam, indicating that PIR foam has a stronger absorption peak at the $1406 \mathrm{~cm}-1$ than that of PU foam, and carbon peak position of PIR foam is shifted to lower wave compared to the PU. It can be seen from the reaction formula (Fig. 3 ) in which the isocyanate is trimerized to form a trimeric structure. Since the carbonyl group in the PIR foam is linked 
to the trimeric structure, it may be due to the conjugation that causes the above changes. Results also indicates that PIR foam contains a large amount of isocyanurate ring structure. The combustion performance of PIR and PU foam can be studied by using cone calorimeter and limit oxygen index. Fig. 4 and Fig. 5 are the heat release rate and smoke release rate of PIR and PU foams, respectively. Tab. 2 shows the combustion performance of the two foams and the combustion performance is mainly compared through the following data, firstly the maximum heat release rate, PU has the maximum heat release rate of $279.8 \mathrm{~kW} / \mathrm{m} 2$, significantly higher than that of the PIR which is $143.6 \mathrm{~kW} / \mathrm{m} 2$, the higher the heat release rate, the better combustion performance of the material, and correspondingly the flame retardant performance is poor. And the total heat release of PU is also higher than the PIR, indicating that combustion of PU is completely than PIR. The release of smoke from the combustion process can also reflect the flame retardancy of the foam, and the amount of smoke released by the PIR in the combustion is less $4.5 \mathrm{~m} 2$ than that of the PU. The final burning carbon residue from PIR foam is also higher than that of the PU foam. In addition, the limiting oxygen index is also a measurement of plastic and other polymer materials relative to the combustion test in order to determine the degree of difficulty in combustion of the material in contact with the flame in air, the higher oxygen index, the greater the flame retardant properties of materials. From Tab. 2, it can be seen that the limit oxygen index of PIR foam is $22 \%$, which is greater than $19 \%$ of PU foam, which further shows that PIR foam has better flame retardancy than PU foam. In summary, it can be seen that the presence of trimeric structures can improve the flame retardancy of PIR foams.

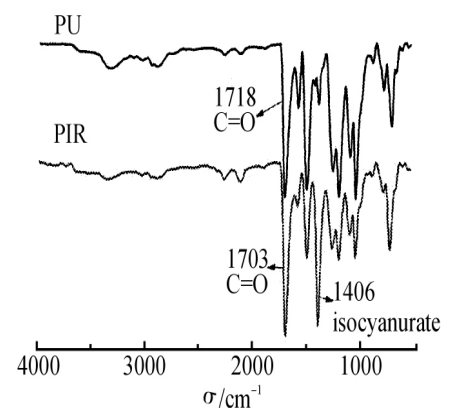

Fig. 2

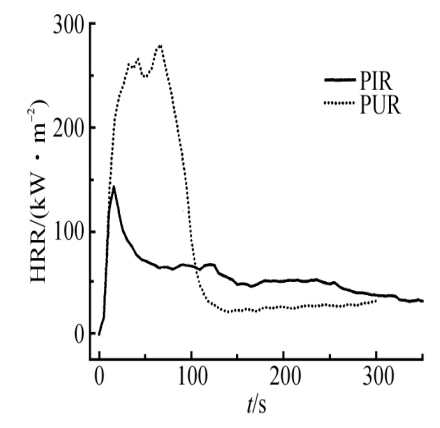

Fig. 4<smiles></smiles>

Fig. 3

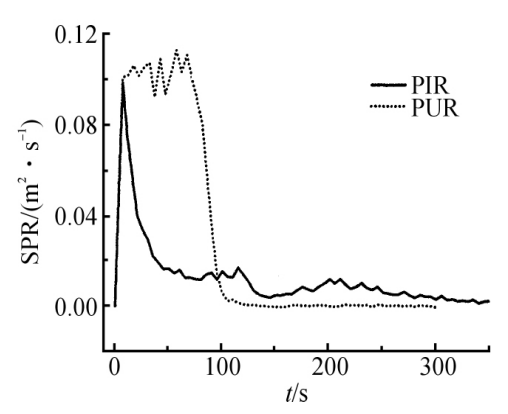

Fig. 5

\begin{tabular}{|l|l|l|l|l|l|}
\hline Sample & HRR $/(\mathrm{kW} \cdot \mathrm{m}-2)$ & THR $/(\mathrm{MJ} \cdot \mathrm{m}-2)$ & TSP $/ \mathrm{m} 2$ & Char residue $/ \%$ & LOI $/ \%$ \\
\hline PU & 279.8 & 26.8 & 8.7 & 14.6 & 19 \\
\hline PIR & 143.6 & 22.8 & 4.2 & 29.9 & 22 \\
\hline
\end{tabular}

Tab. 2

\subsection{The mechanism of thermal decomposition stage in condensed phase}

The mechanism action of trimer structure in increasing the flame retardancy of PIR was studied, analyzed and discussed in 3 different part as mentioned in the following. Firstly, in the mechanism of the thermal decomposition stage in condensed phase, which mainly to study the rate of residual decomposition and to understanding the segments of decomposition at different temperatures by using thermogravimetric analysis and infrared analysis. Fig. 6 and Fig. 7 shows that, in the atmospheric air condition, both PIR and PU foam show two significant heat loss stages, the significant heat loss peak fall at $330{ }^{\circ} \mathrm{C}$ and $540{ }^{\circ} \mathrm{C}$, respectively. As shown in Tab. 3, the decomposition residual rate of PIR at $330{ }^{\circ} \mathrm{C}$ and $540{ }^{\circ} \mathrm{C}$ was significantly higher than that of PU. Compared with PU, the decomposition residual rate of PIR 
increased from $65.8 \%$ to $82.7 \%$ at $330{ }^{\circ} \mathrm{C}$, whereas the rate increased from $18.8 \%$ to $31.7 \%$ at $540{ }^{\circ} \mathrm{C}$. It indicate that the trimer structure has high thermal stability and is not easily decomposed at high temperature, thus it slows down the thermal decomposition process during the combustion of the foam and increase the flame retardancy of the foam. The infrared analysis curves of polyurethane foams from different temperatures are shown in Fig. 8: (1) at room temperature, $1703 \mathrm{~cm}-1$ is the stretching vibration peak for $\mathrm{C}=\mathrm{O}, 1597 \mathrm{~cm}-1$ and $1513 \mathrm{~cm}-1$ is vibration peak of the benzene ring skeleton, $1406 \mathrm{~cm}-1$ is the peak position of trimer structure, $1223 \mathrm{~cm}-1$ is the $\mathrm{C}-\mathrm{O}$ stretching vibration peaks, $1067 \mathrm{~cm}-$ 1 and $1008 \mathrm{~cm}-1$ are C-O-C ethers stretching vibration peaks, and $816 \mathrm{~cm}-1,755 \mathrm{~cm}-1$ and $711 \mathrm{~cm}-1$ are $\mathrm{C}-\mathrm{H}$ outer swing characteristic peaks for para, ortho and meta respectively in the aromatic hydrocarbon structure, all infrared characteristic peaks above are the classical peaks for PIR foam. (2) The infrared characteristic peaks are significantly weakened at $330{ }^{\circ} \mathrm{C}$, including $\mathrm{C}-\mathrm{O}$ and $\mathrm{C}-\mathrm{O}-\mathrm{C}$ were significantly decreased or disappeared, this shows that the first heat loss stage was mainly the fracture and decomposition of the carbamate structure, while the characteristic peak intensity of the trimer structure at this stage was still significant stronger, these indicating that the thermostability of the trimer structure is high at this temperature stage; (3) at $540{ }^{\circ} \mathrm{C}$, the infrared curve is more smooth, only at $1578 \mathrm{~cm}-1$ is the benzene ring $\mathrm{C}=\mathrm{C}$ vibration peak and at $1214 \mathrm{~cm}-1$ is the $\mathrm{C}-\mathrm{O}$ stretching vibration peak, indicating that most of the structure of this stage of thermal decomposition more thorough, some cross-linked structure tends to carbonize. The above analysis show that the trimer structure enables the PIR foam to have good thermal stability, causing the foam is not easily decomposed at high temperature, slowing down the thermal decomposition process of foam in condensation phase, thus play a flame retardant effect.

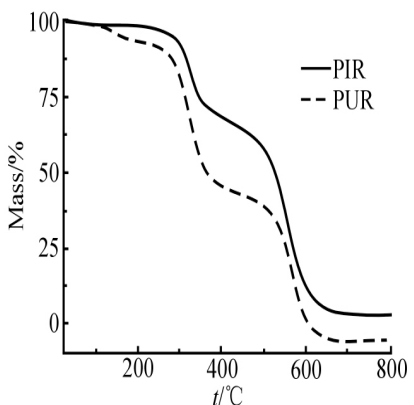

Fig. 6

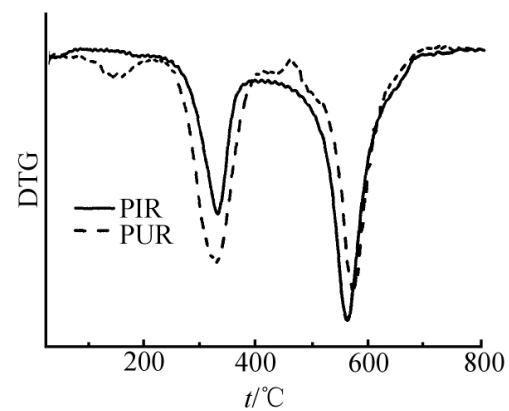

Fig. 7

\subsection{The barrier mechanism of carbon layer formation on the surface in condensed phase}

In order to observe the mechanism of the carbon layer, the optical camera and the scanning electron microscopy (SEM) were used to test on the foam combustion residue which left after the cone calorimeter test. Fig 9 shows the carbon residual decomposition of PU and PIR foam. It is clear that the carbon residue of the PIR foam (Fig.9b) is quite complete, while the carbon residual of PU foam (Fig. 9a) has many holes and cracks. This phenomenon can also be observed more clearly by SEM. Fig. 10 shows that the surface of PU foam residue has many obvious holes, and the structure is more loose. This may be due to the fact that the carbamate segments are easily released through the carbon layer under the action of combustion heat, and causing the more pores and cracks to form on the carbon layer surface. And the PIR foam tends to become charcoal due to the presence of a large number of isocyanurate rings during combustion, and the cross-linked isocyanurate structure is the primary factor in the formation of carbon, resulting in the formation of a dense and firm carbon layer in PIR foams after combustion. In addition, results of the cone calorimeter test shows that the presence of the trimer structure increased the carbon residue after the combustion of the foam, it greatly increased from $14.7 \%$ in PU to $29.9 \%$ in PIR. In summarize, the results show that the trimer structure cause the PIR foam to form a more dense carbon layer than the PU during combustion, and it effectively blocking the oxygen and heat, thus to increase the flame retardancy of the PIR foam.
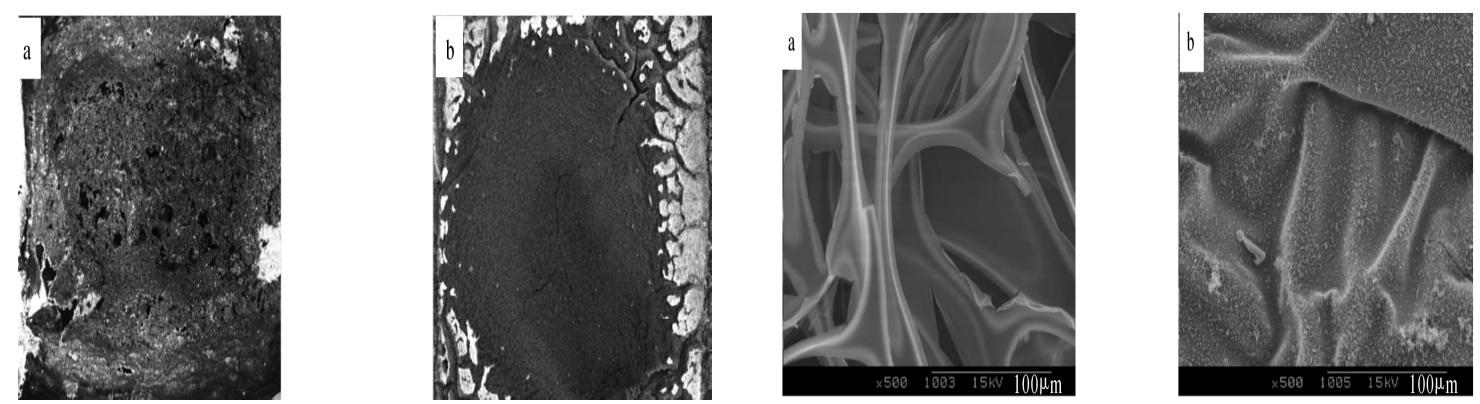

Fig 9

Fig 10 


\subsection{The mechanism in gaseous phase.}

In this paper, the gaseous composition of thermal decomposition in PIR and PU foam was demonstrated by using pyrolysis gas chromatography mass spectrometry, and the mechanism of flame retardation in gaseous was explored. The foam combustion surface temperature is about $600{ }^{\circ} \mathrm{C}$ tested by using temperature measurement probe. The analysis of the carbon layer in previous experiment was also assumed at $600{ }^{\circ} \mathrm{C}$. Therefore, the test conditions of Py / GC-MS were set at $600{ }^{\circ} \mathrm{C}$ and the helium atmosphere is the gaseous composition used to simulate the real combustion. According to the mechanism of gas-phase flame retardancy, the high temperature pyrolysis experiment is mainly analyzed the content of decomposed inert gas $\mathrm{CO} 2$ composition, including the content of combustible gas. Fig 11 shows high temperature pyrolysis gas chromatogram of PIR foam at $600{ }^{\circ} \mathrm{C}$ and Fig. 12 shows the corresponding high temperature pyrolysis mass spectrometry of PIR foam at $600{ }^{\circ} \mathrm{C}$, integrated to qualitative and quantitative analyze the gas composition. Fig 13 and Fig. 14 show the finding of the high temperature pyrolysis gas chromatographic and mass spectrometric analysis of PU samples. Since different peaks correspond to different thermal decomposition compounds, the changes in CO2 content is determined by comparing the surface area of the different peaks. Partial decomposition structure of phthalic anhydride in phthalic acid polyester polyol found to be stay at peak b correspond at the time of 18.936 min in PIR and at $18.978 \mathrm{~min}$ in PU due to the content of the two in the formula is the same, and the structure is more stable, thus it is selected as the reference peak. Peak a of CO2 were found to be at $2.244 \mathrm{~min}$ in the PIR and in the PU was 2.197 min. In addition, the content changes of flammable gas polyol peak c (residence time at $10.883 \mathrm{~min}$ and $10.917 \mathrm{~min}$, respectively) was also investigated.

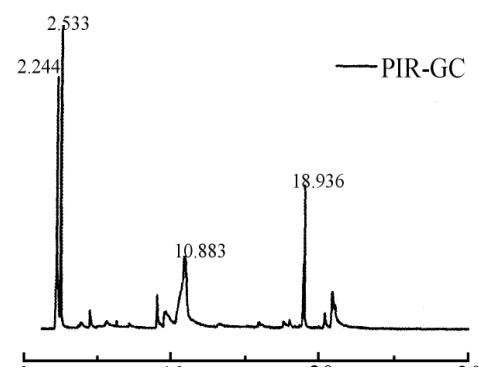

Fig 11

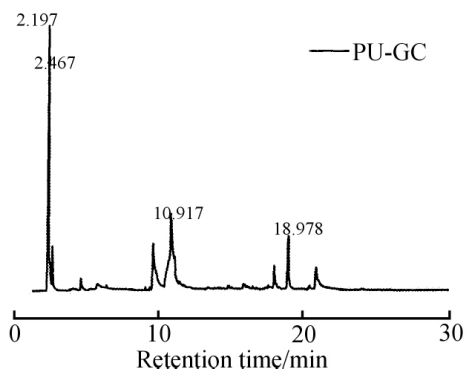

Fig 13

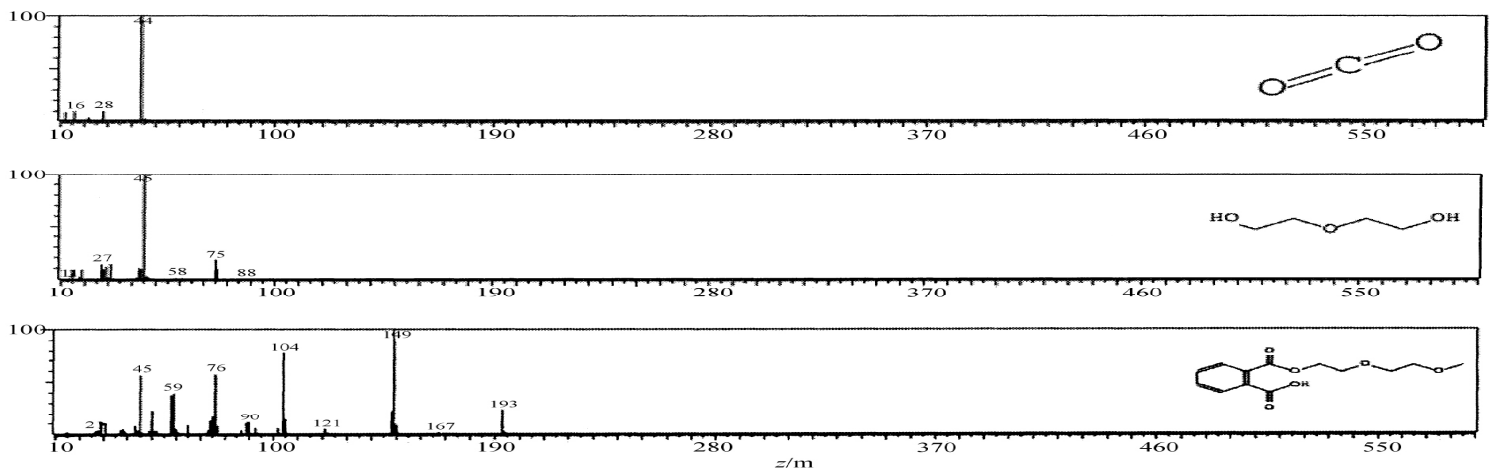

Fig 12
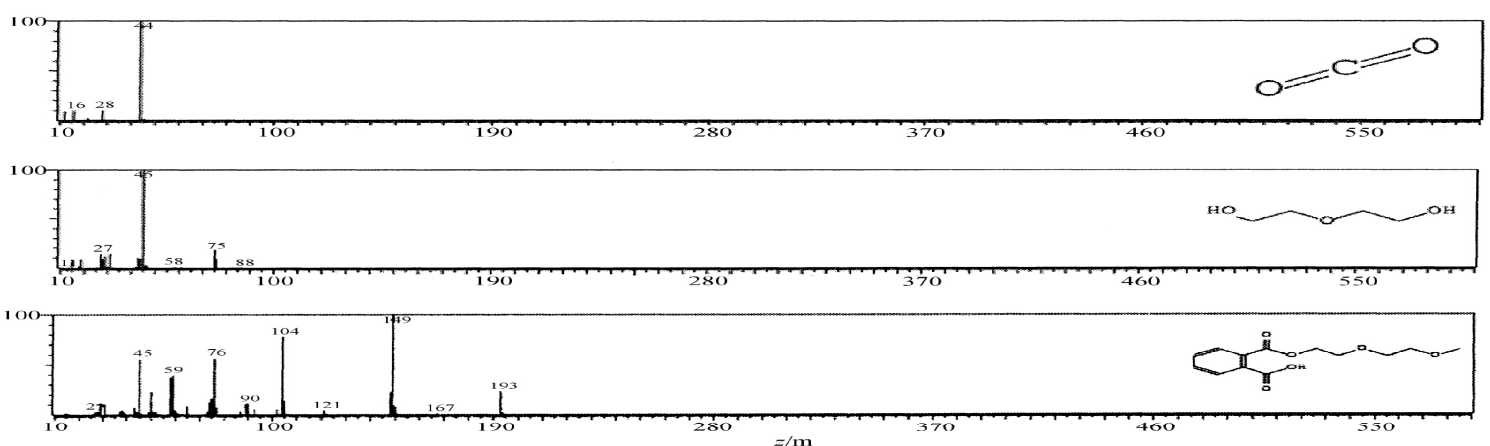

Fig 14 
Data in Tab. 4 shows the integration and comparison of different peaks and peaks area, respectively. Statistics show that the CO2 content in the PIR foam decomposition in gas phase is $79.3 \%$ higher than that of the PU, and the flammable gas polyol is reduced by $36.4 \%$. These indicates that th00e trimer structure can effectively increase the content of inert gas in the gas phase, dilute the flammable gas concentration, and also reduce the flammable gas content in the gas phase, thus improving the flame retardancy of the PIR foam.

\section{Conclusion}

In this paper, the trimer structure is proved to induce flame retardancy effect in PIR foam by using infrared, thermal analysis and high temperature pyrolysis gas chromatography.

(1) TG test results show two biggest heat loss stage and decomposition residual formed at $600{ }^{\circ} \mathrm{C}$. PIR foam have a higher residual rate compared to PU foam and this demonstrating that the trimer cause the PIR foam have higher heat stability. The high temperature infrared test structure indicates that the trimer structure is more thermally stable than the carbamate structure, and it needs to absorb more heat when decomposing, causing the foam is not easily decompose at high temperature and slower the thermal decomposition process in condensed phase and thus induced flame retardant effect.

(2) By observing the morphology of the carbon layer, it can be seen that the trimer structure favors the formation of charcoal and the residual carbon content from $14.7 \%$ of PU to $29.9 \%$ of PIR and causing the PIR foam to form more dense carbon layer, and effectively blocking the oxygen and heat in the combustion, thereby enhancing the flame retardant properties of the foam.

(3) Thermal decomposition of polyurethane in gas phase was tested using Py / GC-MS. The results show that the trimer in the PIR reduces the release of the flammable polyol gas by $36.4 \%$ relative to the PU foam, while also increasing the content of the inert gas carbon dioxide by $79.3 \%$, further supporting the trimer induced flame retardant effect in gas phase.

\section{Reference}

1. Chen Jun. Polyisocyanate Foam [J]. Plastics, 1989, 18 (2): 4-10.Chen J. Polyisocyanurate foam plastics [J]. Plastics, 1989, 18(2): 4-10.

2. Tang Minging, Zhou Qiuming, Tian Chunrong. Polyurethane Modified Polyisocyanurate Foam Heat Resistance Can study [J]. Plastics Industry, 2010,38 (10): 17-20.Tang M J, Zhou Q M, Tian C R. Study on the heat resistance of PU-PIR foam [J]. China Plastics Industry, 2010,38 (10): 17-20.

3. Zhang Jun, Ji Kuijiang, Xia Yanzhi. Polymer combustion and flame retardant technology [M]. Beijing: Chemistry Industrial Press, 2005.

4. Zhang Y, Xia Z, Huang H, Chen H. Thermal degradation Polyurethane based on IPDI [J]. J. Anal. Appl. , 2009, 84: 89-94.

5. Zhang Y, Xia Z, Huang H, Chen H. A degradation study of Waterborne polyurethane based on TDI [J]. Polym. Test. , 2009,28: 264-269.

6. Kulesza K, Pielichowski K, German K. Thermal decompositionOf bisphenol A-based polyetherurethanes blown with pentane:Part I-Thermal and pyrolytical studies [J]. J. Anal. Appl. 2006, 76: 243-248.

7. Kunze R, Schartel B, Bartholmai M, et al. TG-MS and TG FIIR applied for anunambiguous thermal analysis of intumescent Coatings [J]. J. Therm. Anal. Calorim. , 2002, 70: 897-909.

8. $\mathrm{Hu} \mathrm{X}$ M, Wang D M, Wang S L. Synergistic effects of expandable Graphite and dimethyl methyl phosphonate on the mechanical Properties, fire behavior, and thermal stability of a polyisocyanurate- Polyurethane foam International [J]. J. Min. Sci. Technol. ,2013,23: 13-20.

9. Gao L P, Zheng G Y, Zhou Y H, et al. Synergistic effect Expandable graphite, diethylethylphosphonate and organicallymodified Layered double hydroxide on flame retardancy and fire Behavior of polyisocyanurate-polyurethane foam nanocomposite[J]. Polym. Degrad. Stab., 2014, 101: 92-101.

10. Modesti M, Lorenzetti A. An experimental method for comparison Isocyanate conversion and trimer formation in polyisocyanate-Polyurethane foams [J]. Eur. Polym. J. , 2001, 37: 949-954. 